Supporting Information for

\title{
Retrieving the Rate of Reverse Intersystem Crossing from Ultrafast Spectroscopy
}

Jiahua Hu, Qun Zhang*, and Yi Luo*

Hefei National Laboratory for Physical Sciences at the Microscale, Department of Chemical Physics, Synergetic Innovation Center of Quantum Information and Quantum Physics, University of Science and Technology of China, Hefei, Anhui 230026, P. R. China

*All correspondence should be addressed to

Q. Zhang (qunzh@ustc.edu.cn) or Y. Luo (yiluo@ustc.edu.cn). 


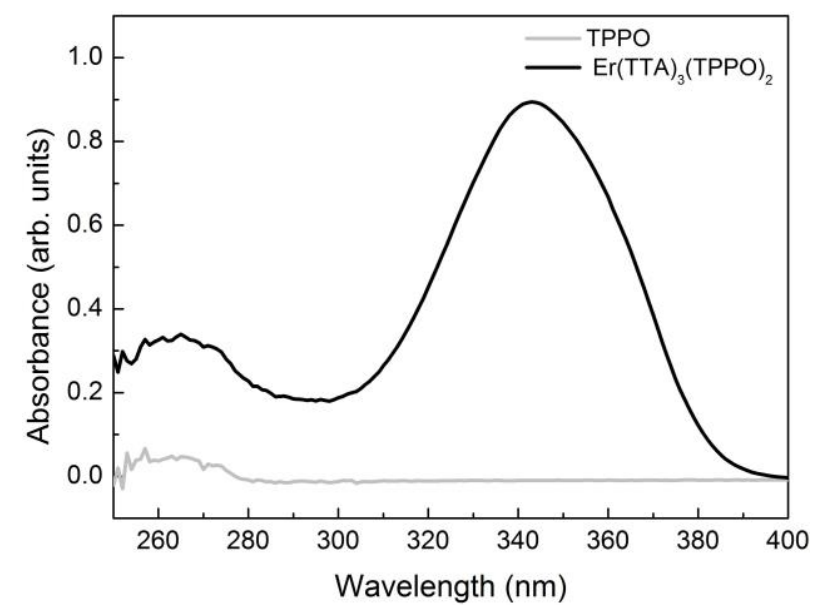

Figure S1. Steady-state absorption spectra recorded on the investigated $\operatorname{Er}(\mathrm{TTA})_{3}(\mathrm{TPPO})_{2}$ sample $(15 \mu \mathrm{mol} / \mathrm{L}$, well dissolved in DMSO). The predominant band peaking at around $340 \mathrm{~nm}$ corresponds to the $S_{0} \rightarrow S_{1}$ transition in TTA, from which the bottom of the $S_{1}$ state can be estimated to be $\sim 25000 \mathrm{~cm}^{-1}$ (i.e., $\sim 400 \mathrm{~nm}$ ) relative to that of the $S_{0}$ state. Note that the synergetic ligand molecule TPPO shows nearly no absorption in the UV region around $340 \mathrm{~nm}$ (see the spectral profile in light grey, which was recorded also in the concentration of $15 \mu \mathrm{mol} / \mathrm{L}$ in DMSO). 

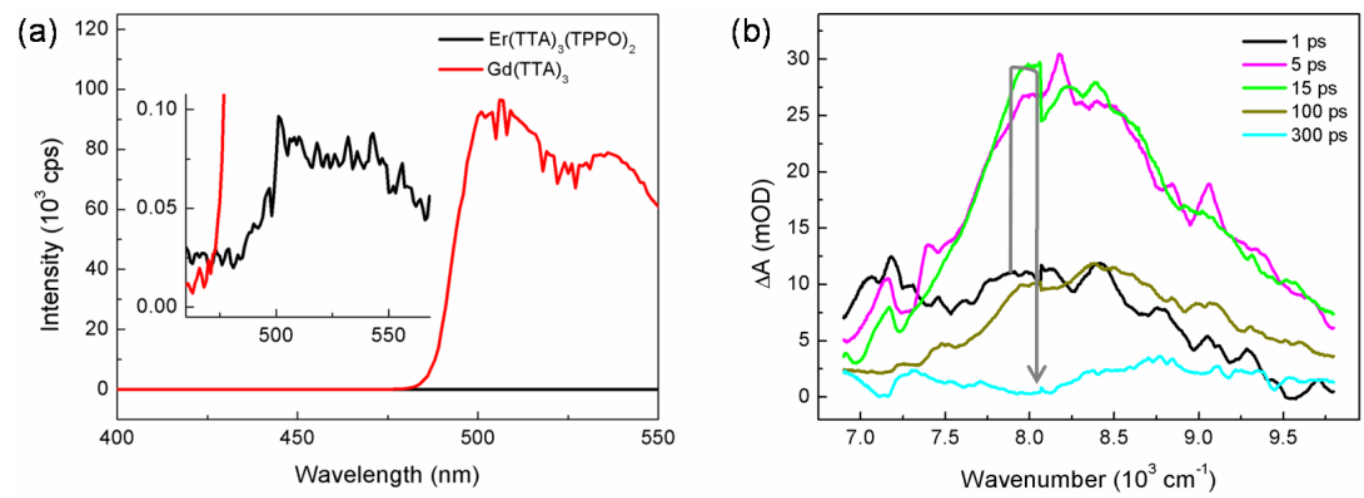

Figure S2. (a) Determination of the $\mathrm{T}_{1}$-state energetics with the assistance of phosphorescence spectra measured on the $\operatorname{Er}(\mathrm{TTA})_{3}(\mathrm{TPPO})_{2}$ sample and a reference sample of $\mathrm{Gd}(\mathrm{TTA}) 3$. As the $\mathrm{Er}^{3+}$ chelate molecules (such as the $\operatorname{Er}(\mathrm{TTA})_{3}$ investigated in this work) usually feature a relatively fast resonant energy transfer (RET) process (on a time scale of sub-100 ps for $\operatorname{Er}(\mathrm{TTA})_{3}$ ), their phosphorescence emissions are rather weak. As such, the rare-earth photoluminescence community usually uses a certain alternative molecule that strongly phosphoresces (such as the well-known Gd(TTA) 3 used here) to help identify the triplet-state energetics involved. As for the current $\operatorname{Er}(\mathrm{TTA})_{3}$ system, the bottom of the $\mathrm{T}_{1}$ state was thus determined to be $\sim 20500 \mathrm{~cm}^{-1}$ (i.e., $\sim 488 \mathrm{~nm}$ ) relative to that of the $S_{0}$ state, in agreement with a documented value from the phosphorescence measurement on $\operatorname{Gd}(\mathrm{TTA})_{3} .{ }^{\mathrm{S} 1}$ Representative transient absorption spectra recorded in the near infrared region $1020-1450 \mathrm{~nm}$ (i.e., $\sim 6900-9800 \mathrm{~cm}^{-1}$ ) at probe delays of 1, 5, 15, 100, and 300 ps, from which one can detect a similar evolution of spectral profiles to that in Band-A (Figure 2a, main text) [refer to the grey arrow that guides the eye], indicating excited-state absorption from $\mathrm{T}_{1}$ to a certain high-lying excited triplet state $T_{n}$. On the basis of this observation along with the above mentioned $T_{1}$-state energetics, the bottom of this $T_{n}$ state can be estimated to locate at around $27400 \mathrm{~cm}^{-1}$, as schematically depicted in Figure 1 (main text). 


\section{Supplementary Note}

Presented here are the details of modeling formulation that starts with eqs 1.1 and 1.2 (main text) and ends with eqs 2.1 and 2.2 (main text).

$$
\begin{aligned}
& \frac{\partial S(t)}{\partial t}=-k_{\mathrm{ISC}} S(t)+k_{\mathrm{rISC}} T(t) \\
& \frac{\partial T(t)}{\partial t}=k_{\mathrm{ISC}} S(t)-k_{\mathrm{rISC}} T(t)-k_{\mathrm{RET}} T(t)
\end{aligned}
$$

\{eq 1.1, main text $\}$

$\{$ eq 1.2, main text $\}$

For simplicity, let $S(t)=x, T(t)=y, k_{\mathrm{ISC}}=k_{1}, k_{\mathrm{rISC}}=k_{2}$, and $k_{\mathrm{RET}}=k_{3}$, then we have

$$
\begin{aligned}
& \frac{\mathrm{d} x}{\mathrm{~d} t}=-k_{1} x+k_{2} y \\
& \frac{\mathrm{d} y}{\mathrm{~d} t}=k_{1} x-k_{2} y-k_{3} y
\end{aligned}
$$

The initial conditions are $y(0)=0$ and $x(0)=1$. By eliminating $y$, we have

$$
\frac{\mathrm{d}^{2} x}{\mathrm{~d} t^{2}}+\left(k_{1}+k_{2}+k_{3}\right) \frac{\mathrm{d} x}{\mathrm{~d} t}+k_{1} k_{3}=0
$$

The corresponding characteristic equation is

$$
z^{2}+\left(k_{1}+k_{2}+k_{3}\right) z+k_{1} k_{3}=0
$$

Its solution takes the form of

$$
z=\frac{-\left(k_{1}+k_{2}+k_{3}\right) \pm \sqrt{\left(k_{1}+k_{2}+k_{3}\right)^{2}-4 k_{1} k_{3}}}{2}
$$

The expression in the square root can be rearranged as

$$
\left(k_{1}+k_{2}+k_{3}\right)^{2}-4 k_{1} k_{3}=\left(k_{1}+k_{2}-k_{3}\right)^{2}+4 k_{2} k_{3}
$$

Under proper approximations $k_{1}>k_{2}$ and $k_{1}>>k_{3}$ (validation discussed at the end of this Note), we have

$$
\sqrt{\left(k_{1}+k_{2}+k_{3}\right)^{2}-4 k_{1} k_{3}}=\sqrt{\left(k_{1}+k_{2}-k_{3}\right)^{2}+4 k_{2} k_{3}} \approx\left(k_{1}+k_{2}-k_{3}\right)+\frac{2 k_{2} k_{3}}{\left(k_{1}+k_{2}-k_{3}\right)}
$$

Thus the solutions for $z$ read 


$$
\begin{aligned}
& z_{1}=\frac{-\left(k_{1}+k_{2}+k_{3}\right)+\left(k_{1}+k_{2}-k_{3}\right)+\frac{2 k_{2} k_{3}}{\left(k_{1}+k_{2}-k_{3}\right)}}{2}=-\frac{k_{1} k_{3}-k_{3}^{2}}{\left(k_{1}+k_{2}-k_{3}\right)} \\
& z_{2}=\frac{-\left(k_{1}+k_{2}+k_{3}\right)-\left[\left(k_{1}+k_{2}-k_{3}\right)+\frac{2 k_{2} k_{3}}{\left(k_{1}+k_{2}-k_{3}\right)}\right]}{2}=-\left[\left(k_{1}+k_{2}\right)+\frac{k_{2} k_{3}}{\left(k_{1}+k_{2}-k_{3}\right)}\right]
\end{aligned}
$$

and the general solutions for $x$ and $y$ read

$$
\begin{gathered}
x=c_{1} \exp \left[-\frac{k_{1} k_{3}-k_{3}^{2}}{\left(k_{1}+k_{2}-k_{3}\right)} t\right]+c_{2} \exp \left\{-\left[\left(k_{1}+k_{2}\right)+\frac{k_{2} k_{3}}{\left(k_{1}+k_{2}-k_{3}\right)}\right] t\right\} \\
k_{2} y=c_{1}\left[k_{1}-\frac{k_{1} k_{3}-k_{3}^{2}}{\left(k_{1}+k_{2}-k_{3}\right)}\right] \exp \left[-\frac{k_{1} k_{3}-k_{3}^{2}}{\left(k_{1}+k_{2}-k_{3}\right)} t\right]+ \\
c_{2}\left[-k_{2}+\frac{k_{2} k_{3}}{\left(k_{1}+k_{2}-k_{3}\right)}\right] \exp \left\{-\left[\left(k_{1}+k_{2}\right)+\frac{k_{2} k_{3}}{\left(k_{1}+k_{2}-k_{3}\right)}\right] t\right\}
\end{gathered}
$$

By noting that $y(0)=0$, we have

$$
c_{1}\left[k_{1}-\frac{k_{1} k_{3}-k_{3}^{2}}{\left(k_{1}+k_{2}-k_{3}\right)}\right]+c_{2}\left[-k_{2}+\frac{k_{2} k_{3}}{\left(k_{1}+k_{2}-k_{3}\right)}\right]=0
$$

Considering that $k_{1}>>k_{3}$, we have

$$
\begin{aligned}
& c_{1} k_{1}-c_{2} k_{2} \approx 0 \\
& z_{1}=-\frac{k_{1} k_{3}-k_{3}^{2}}{\left(k_{1}+k_{2}-k_{3}\right)} \approx-\frac{k_{1} k_{3}}{k_{1}+k_{2}} \\
& z_{2}=-\left[\left(k_{1}+k_{2}\right)+\frac{k_{2} k_{3}}{\left(k_{1}+k_{2}-k_{3}\right)}\right] \approx-\left(k_{1}+k_{2}\right)
\end{aligned}
$$

Let $\kappa=k_{2} / k_{1}$, then we have

$$
\kappa=c_{1} / c_{2}
$$

By noting that $x(0)=c_{1}+c_{2}=1$, we have

$$
\begin{aligned}
& c_{1}=\frac{\kappa}{1+\kappa} \\
& c_{2}=\frac{1}{1+\kappa}
\end{aligned}
$$

Taken together, the above results lead to 


$$
\begin{aligned}
& x=\frac{1}{1+\kappa} \exp \left[-(1+\kappa) k_{1} t\right]+\frac{\kappa}{1+\kappa} \exp \left(-\frac{1}{1+\kappa} k_{3} t\right) \\
& y=-\frac{1}{1+\kappa} \exp \left[-(1+\kappa) k_{1} t\right]+\frac{1}{1+\kappa} \exp \left(-\frac{1}{1+\kappa} k_{3} t\right)
\end{aligned}
$$

i.e., we finally arrive at eqs 2.1 and 2.2 in the main text

$$
\begin{array}{ll}
S(t)=\frac{1}{1+\kappa} \exp \left[-(1+\kappa) k_{\mathrm{ISC}} t\right]+\frac{\kappa}{1+\kappa} \exp \left(-\frac{1}{1+\kappa} k_{\mathrm{RET}} t\right) & \text { \{eq 2.1, main text }\} \\
T(t)=-\frac{1}{1+\kappa} \exp \left[-(1+\kappa) k_{\mathrm{ISC}} t\right]+\frac{1}{1+\kappa} \exp \left(-\frac{1}{1+\kappa} k_{\mathrm{RET}} t\right) & \{\text { eq 2.2, main text }\}
\end{array}
$$

It is worth mentioning that the approximations (i.e., $k_{\mathrm{ISC}}>k_{\mathrm{rISC}}$ and $\left.k_{\mathrm{ISC}}>>k_{\mathrm{RET}}\right)$ in the above formulation are adopted simply for the sake of explicitly deducing analytical expressions with clear physical meaning. Such proper approximations have been well justified for the current case study (and also for many other similar rare-earth chelate systems). Refer to the relevant fitting results based on our ultrafast spectroscopy measurements (main text): $k_{\mathrm{ISC}} \approx 0.235 \mathrm{ps}^{-1}, k_{\mathrm{rISC}} \approx 0.068 \mathrm{ps}^{-1}$, and $k_{\mathrm{RET}} \approx 0.017 \mathrm{ps}^{-1}$, which obviously validate the two relations in the adopted approximations. 


\section{Supplementary References}

(S1) Sager, W. F.; Filipescu, N.; Serafin, F. A. Substituent effects on intramolecular energy transfer. I. Absorption and phosphorescence spectra of rare earth $\beta$-diketone chelates. J. Phys. Chem. 1965, 69, 1092-1100. 